\title{
Commentary on urinary incontinence and chronic conditions in the US population age $\mathbf{5 0}$ years and older
}

\author{
Radhika Patnam ${ }^{1}$ \\ Received: 6 October 2019 / Accepted: 17 October 2019/Published online: 26 November 2019 \\ (C) The International Urogynecological Association 2019
}

The purpose of this study is to examine the association of urinary incontinence with chronic conditions in the US population using the National Health and Nutrition Examination survey (NHANES). The US population is aging, and incontinence symptoms are becoming a larger burden, but are often underreported by patients and thus not treated. Incontinence may play a role in management of other chronic conditions or assessing daily needs for elderly patients.

The NHANES database was used focusing specifically on those $>50$ years of age with defined race who completed questions regarding incontinence. A total of 7226 women and 7239 men were included in the analysis. Chronic conditions were self-reported. Prevalence of mixed incontinence (MUI) was higher in the presence of diabetes, hypertension, heart failure, and CVD. No significant association was noted with diuretics or beta-blockers in men or women. Both heart failure (HF) and diabetes mellitus (DM) were associated with higher odds of MUI in women.

In upcoming years with an increasing elderly population, understanding the interaction of complex chronic disease with incontinence will only help patients improve their quality of life. This study helps to see that many chronic conditions, such as HF and DM, are associated with MUI. However, these data are all self-reported, making it difficult to interpret, especially as these diseases can be well controlled. More understanding of the impact MUI may have on treatment expectations or adherence can help to better serve patients with chronic disease.

Publisher's note Springer Nature remains neutral with regard to jurisdictional claims in published maps and institutional affiliations.
Radhika Patnam

radhika_patnam@med.unc.edu

1 University of North Carolina at Chapel Hill, Chapel Hill, NC, USA 